\title{
Pyoderma Gangrenosum Following the Revision of a Breast Reconstruction and Abdominoplasty
}

\author{
Mio Nakamura ${ }^{*}, 1$, Amir M. Ghaznavi², Vigen Darian ${ }^{2}$ and Aamir Siddiqui ${ }^{2}$ \\ ${ }^{1}$ Wayne State University School of Medicine, Detroit, MI, USA \\ ${ }^{2}$ Division of Plastic and Reconstructive Surgery, Department of Surgery, Henry Ford Health System Detroit, MI, USA
}

\begin{abstract}
Pyoderma gangrenosum (PG) is a rare ulcerative dermatologic disease and little is known about its etiology and pathogenesis. Recent reports show that there have been limited but increasing number of cases of PG following aesthetic surgeries. Post-surgical PG is often misdiagnosed, which can have serious clinical consequences. The following case report describes a young woman who underwent a cosmetic breast augmentation and abdominoplasty which was complicated by post-operative necrotizing fasciitis. She was presented one year later for surgical correction of her acquired breast and abdominal deformities. Post-operatively she developed a severe inflammatory skin response presumed to be a wound infection. However, after repeated surgical debridements, the wounds persisted without a defined bacterial or fungal organism. After clinical exclusion of all other etiologies, PG was diagnosed and confirmed with histopathology. The patient was subsequently treated with aggressive immunosuppressive therapy, and the lesions resolved without any signs of residual PG. This case report attempts to increase awareness for the rare post-surgical complication of PG in aesthetic surgery and to improve future diagnosis and management of such cases.
\end{abstract}

Keywords: Abdominoplasty, breast reconstruction, neutrophilic dermatoses, post-operative complication, pyoderma gangrenosum, wound complication.

\section{INTRODUCTION}

Pyoderma gangrenosum (PG) is a rare ulcerative dermatologic disease in which little is known about its etiology and pathogenesis. $\mathrm{PG}$ is characterized by inflammatory skin lesions without evidence of infection. PG is commonly associated with systemic diseases such as inflammatory bowel disease, hematologic disorders, arthritis, and psoriasis, suggesting a possible autoimmune process. When manifesting outside of this context, PG can go largely unrecognized. An extensive Medline search from 1980-2012 shows that there have been limited but increasing number of reported cases of $\mathrm{PG}$ as a post-operative complication following aesthetic surgeries [1-3]. Pathergy, a process in which cutaneous tissue antigens become altered or exposed following trauma and subsequently become vulnerable to host-mediated immune responses, is thought to be involved [4].

Post-surgical PG is often misdiagnosed as a wound infection, which can have serious clinical consequences. Clinical manifestations of PG vary and contribute to the difficulty of diagnosis. Its similarity in presentation to necrotizing fasciitis and other soft tissue infections can delay diagnosis of PG [5]. The following case report attempts to increase awareness for the rare post-surgical complication of PG in aesthetic surgery and to improve future diagnosis and management of such cases.

*Address correspondence to this author at the Wayne State University School of Medicine, 540 E. Canfield St., Detroit, MI 48201, USA;

Tel: 313-577-1463; Fax: 313-577-0361;

E-mail: mnakamur@med.wayne.edu

\section{CASE REPORT}

A 31-year-old female presented in septic shock at postoperative day 13 following a bilateral breast augmentation, mastopexy, and abdominoplasty. She was promptly taken for debridement of the infected surgical sites and removal of the implants. She underwent a prolonged hospital course undergoing a series of debridements and washouts of the wounds. Over the course of five months the surgical wounds healed.

She returned one year later with a chronic draining sinus from her abdomen and an acquired deformity to her abdominal and chest walls. She requested operative revision of the deformities and excision of the sinus tract (Fig. 1). The procedure entailed repositioning the umbilicus, removing the abdominal wall scarring and sinus tract, and concentric resection of skin around the nipple-areolar complex bilaterally. There were no operative complications, and the patient was discharged from the hospital a few days following the operation.

She returned two days following discharge with fever and bloody drainage from her abdominal incision. She was mildly tachycardic with acute blood loss anemia. She was taken for evacuation of a $250 \mathrm{cc}$ hematoma. The wound was left open and managed post-operatively with negative pressure therapy. She subsequently went for serial debridements of the abdominal and breast wounds, which continued to appear necrotic with drainage of murky fluid. No microbial organisms could be isolated from any operative cultures or drained fluid; however, she continued to spike fevers and have an elevated white blood cell count. 

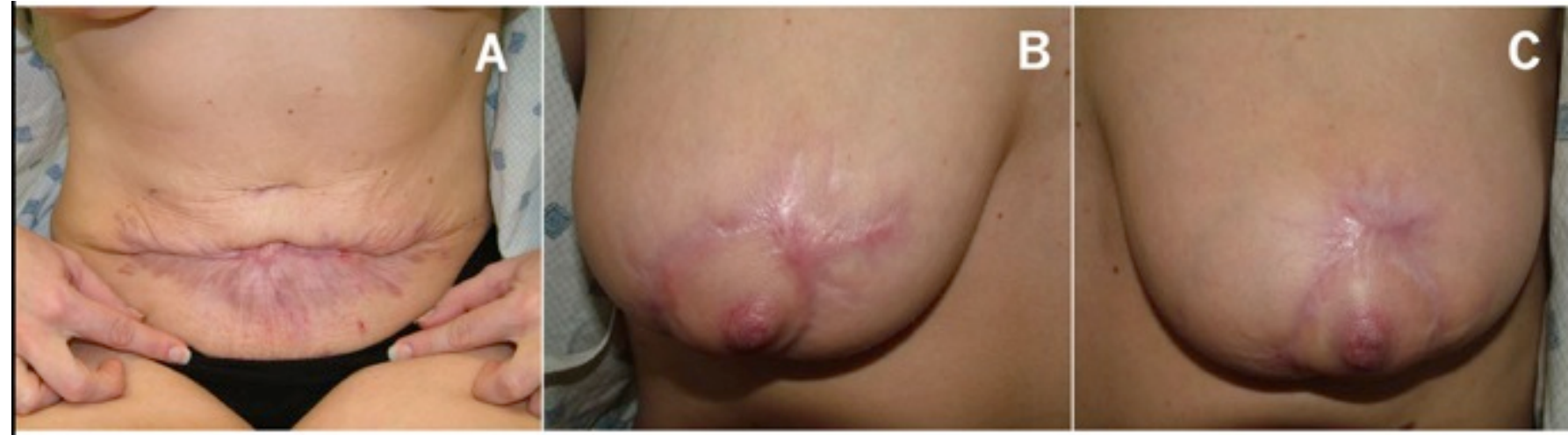

Fig. (1). (A-C). Abdominal sinus tract and contracted breast scars from complications of initial breast reconstruction and abdominoplasty.

Over the course of her hospital stay, two small violacious papules in the suprapubic area developed near the abdominal wound edge. Tissue samples were obtained for histopathology and staining for fungal and bacterial organisms. Histopathology of the tissue demonstrated dense neutrophilic infiltrate consistent with PG (Fig. 2). She was started on immunosuppressive therapy with cyclosporine $175 \mathrm{mg} /$ day $(3 \mathrm{mg} / \mathrm{kg})$. When the tissues stains came back negative for fungal and bacterial organisms, the cyclosporine was increased to $275 \mathrm{mg}$ /day ( $5 \mathrm{mg} / \mathrm{kg}$ ); however, new lesions continued to develop over the next few days. She was subsequently given a dose of infliximab $300 \mathrm{mg}(5 \mathrm{mg} / \mathrm{kg})$. Within 48 hours she became afebrile, the white blood cell count trended down, and her pain decreased significantly. Examination four days later showed some active areas of PG; therefore pulse solumedrol $1 \mathrm{~g}$ /day for five days was added. After completing the regimen, she was started on oral prednisone $60 \mathrm{mg}$ /day while on cyclosporine $275 \mathrm{mg} /$ day. At discharge from the hospital, the areas of PG were almost completely re-epithelialized without new active areas (Fig. 3). She was discharged home on cyclosporine $175 \mathrm{mg} /$ day and an eight-day taper dose of prednisone.

During the course of five months, skin grafts were placed over all wounds. There were no residual sign of PG. She was seen at a two-year post-operative visit with complete resolution of PG and well healed scars of her breast and abdomen (Fig. 4).

\section{DISCUSSION}

It has been eighty years since Brunsting made the first description of PG in 1930 [6], yet little progress has been
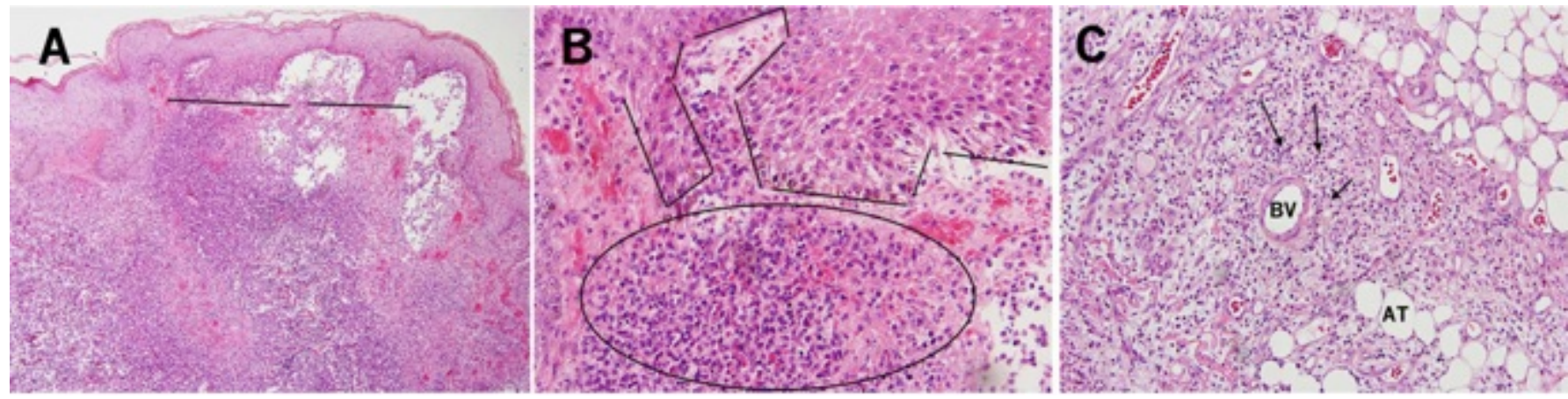

Fig. (2). (A) Hematoxylin and eosin stain of ulcerations, with disruption of the dermal-epidermal junction (black lines), 40x; (B) Disruption of dermal epidermal junction (black lines) with an aggregation of neutrophils in the dermis with infiltration into the epidermis (black circle), 200x; (C) Aggregation of neutrophils (arrows) surrounding blood vessels (BV) and adipose tissue (AT) 200x.
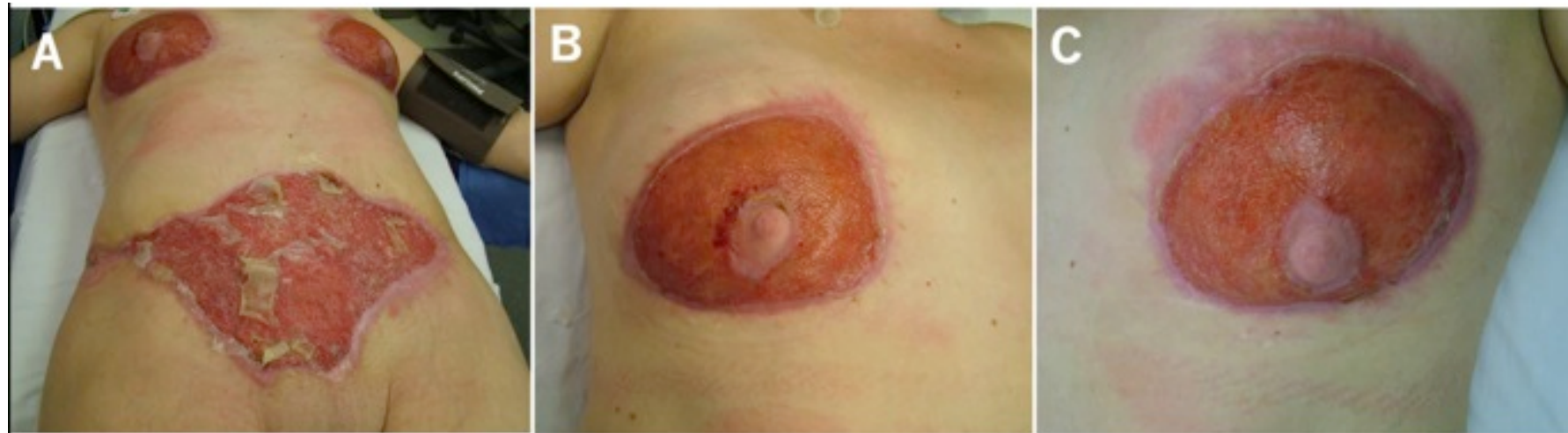

Fig. (3). (A-C). Wounds after initiation of immunosuppressive therapy. 

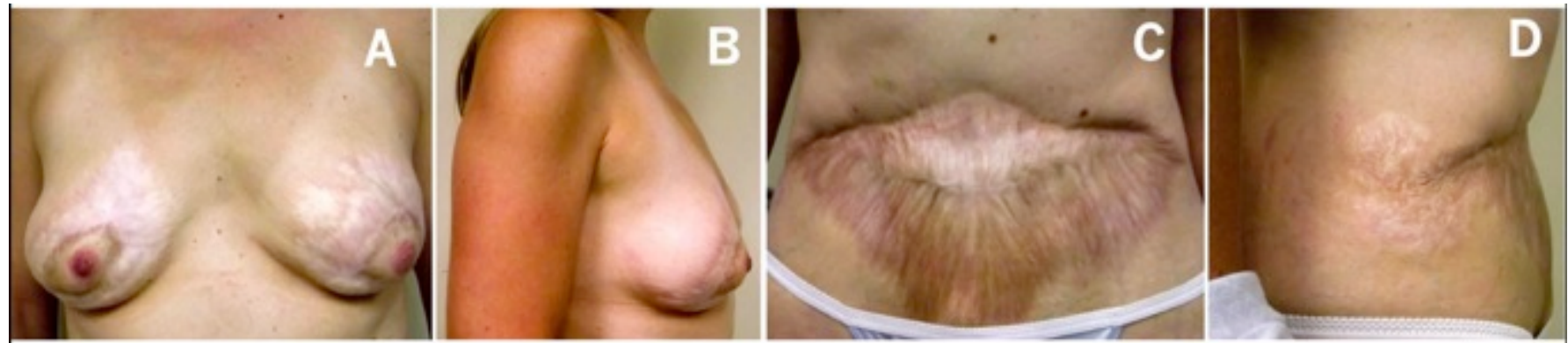

Fig. (4). (A-D). At the two-year post-operative visit with complete resolution of PG and well healed scars of her breast and abdomen.

made in defining the etiology and pathogenesis. There are increasing reports of PG as a post-surgical complication in patients with no known autoimmune diagnosis. Awareness of such a presentation of $\mathrm{PG}$ is lacking, which ultimately leads to significant disfigurement and impairment for the patient.

Inquiring about the patient's medical history will reveal diseases that may be associated with PG, such as inflammatory bowel disease, hematologic disorders, arthritis, and psoriasis. Laboratory values such as antinuclear antibody titer and rheumatoid factor can be useful to rule out possible underlying autoimmune diseases. Other findings are nonspecific, such as leukocytosis and elevated erythrocyte sedimentation rate and C-reactive protein. In our case, the patient had no known history or abnormal labs that could be associated with her subsequent diagnosis of PG.

Histopathology of PG will show nonspecific neutrophilic infiltrate. Wound culture and staining for bacteria and fungi should be negative in PG; however, it is possible to have colonization or secondary infection of PG lesions. In our case, we began to suspect PG when histopathology showed dense neutrophilic infiltrates. At that time, we did not have the final results of the bacterial and fungal staining and therefore could not initiate full treatment with immunosuppressant therapy.

There are many types of post-surgical wound complications, many of which have similar presentations. This contributes to the difficulty in diagnosis and management of post-surgical wound complications. A simple wound infection can develop into more severe soft tissue infections such as necrotizing fasciitis. Skin color will change from red-purple to blue-grey, followed by bullae and gangrene formation. In necrotizing fasciitis, the infection will have spread down to the muscle fascia and can later be anesthetized [7]. Although four variants of PG have been described, PG is most often characterized by rapid development of painful and necrolytic ulcers with irregular, violaceous, and undermined borders [8]. In our case, the patient initially presented with a hematoma. It is unknown whether PG was present at this time or if it manifested following the hematoma evacuation; it is possible that PG does not always present in its classical forms.

Ultimately, clinical diagnosis of PG should be entertained when there is evidence of an overabundant inflammatory response despite the absence of microorganisms on culture or histopathological staining. In PG, repeated irritation of the lesions worsens the condition.
There should be high suspicion for PG when a suspected wound infection spreads or worsens with serial debridemnts.

Once diagnosis of PG is established, prompt treatment is indicated with pain management and local and/or systemic immunosuppressant therapy. Local wound care is important for protecting the site from further trauma. Surgical debridement should be avoided but may not be contraindicated when there is a need for removal of necrotic borders [9]. Although immunosuppressant therapy will quickly arrest progression, providers should be aware that it increases vulnerability to infection [10].

Attempts to increase awareness for post-surgical PG and its complex clinical manifestations are beneficial for decreasing the disease burden. In our case, early consideration of PG would have made a significant difference in the outcome for the patient. Further studies are indicated to discover how to provide a beneficial woundhealing environment for patients with PG on immunotherapy to avoid opportunistic infections. Since PG has a tendency to recur, the role of prophylactic immunosuppressive therapy with future surgical intervention will be a topic of interest for future research.

\section{CONFLICT OF INTEREST}

The authors confirm that this article content has no conflict of interest.

\section{ACKNOWLEDGEMENTS}

We thank Dr. Juan Gomez-Gelvez for his assistance and interpretation of pathology slides.

\section{REFERENCES}

[1] Rajapakse Y, Bunker CB, Ghattaura A, et al. Case report Pyoderma gangrenosum following Deep Inferior Epigastric Perforator (Diep) free flap breast reconstruction. J Plast Reconstruct Anesthet 2010; 63: e395-6.

[2] De Felice E, Bronwen A. Pyoderma gangrenosum; a rare complication of sclerotherapy. Phlebology 2009; 5: 213-4.

[3] Bonamigo RR, Behar PR, Bellar C, Bonfia R. Pyoderma gangrenosum after silicone prosthesis implant in the breasts and facial plastic surgery. Int J Dermatol 2008; 47: 289-91.

[4] Powell FC, Su WP, Perry HO. Pyoderma gangrenosum: a review of 86 cases. Q J Med 1985; 55: 173-86.

[5] Barr KL, Chatwal HK, Wesson SK, Bhattacharyya I, Vincek V. Pyoderma gangrenosum masquerading as necrotizing fasciitis. Am J Otolaryng 2009; 30: 273-6.

[6] Brunsting LA, Goeckerman WH, O'Leary PA. Pyoderma gangrenosum: clinical and experimental observations in five cases occurring in adults. Arch Dermatol and Syph 1930; 22: 655-80.

[7] Schwartz MN, Pasternack MS. Cellulitis and subcutaneous tissue infections. In: Mandell GL, Bennett JE, Dolin R, editors. Principles 
and Practice of Infectious Diseases. 6th ed. Philadelphia: Churchill Livingstone; 2005. p. 1172.

[8] Dabade TS, Davis MDP. Diagnosis and treatment of the neutrophilic dermatoses (pyoderma gagrenosum, Sweet's syndrome). Dermatol ther 2011; 24: 273-84.

[9] Shadt CR, Callen JP. Management of neutrophillic dermatoses. Dermatol ther 2012; 25: 158-72.
[10] Schintler MV, Grohman M, Donia C, Aberer E, Scharnagl E. Management of an unfortunate triad after breast reconstruction: pyoderma gangrenosum, full-thickness chest wall defect and Acinetobacter Baumannii infection. Reconstr Aesthet surg 2010; 63: e564-7.

(C) Nakamura et al.; Licensee Bentham Open.

This is an open access article licensed under the terms of the Creative Commons Attribution Non-Commercial License (http://creativecommons.org/licenses/by-nc/3.0/) which permits unrestricted, non-commercial use, distribution and reproduction in any medium, provided the work is properly cited. 